Revue internationale P.M.E.

Économie et gestion de la petite et moyenne entreprise

\title{
Le transfert de la ressource réseau lors d'un processus de reprise
}

\section{Sébastien Geindre}

Volume 22, numéro 3-4, 2009

URI : https://id.erudit.org/iderudit/044130ar

DOI : https://doi.org/10.7202/044130ar

Aller au sommaire du numéro

\section{Éditeur(s)}

Presses de l'Université du Québec

ISSN

0776-5436 (imprimé)

1918-9699 (numérique)

Découvrir la revue

Citer cet article

Geindre, S. (2009). Le transfert de la ressource réseau lors d'un processus de reprise. Revue internationale P.M.E., 22(3-4), 109-137.

https://doi.org/10.7202/044130ar

\section{Résumé de l'article}

Le transfert de la ressource réseau est-il possible dans le cadre d'une reprise d'entreprise par une personne physique ? Et si oui, comment ? Après avoir présenté les concepts de réseau et de réseautage et montré que ceux-ci peuvent être appréhendés respectivement comme une ressource et une compétence de l'organisation et/ou de son dirigeant dans le cadre d'une petite entreprise, nous cherchons à montrer la difficulté de transférer ces actifs dans un processus repreneurial. À partir de quatre cas exploratoires et d'une étude de cas en profondeur, nous proposons d'associer au processus repreneurial une prise en compte spécifique de la ressource réseau en quatre étapes (perception, évaluation, utilisation, valorisation). La prise en main effective de la ressource réseau gérée par le cédant pouvant donner lieu à trois types de valorisation de la ressource : l'appropriation (le repreneur absorbe en l'état la ressource réseau et a la capacité de la mobiliser) ; l'adjonction (c'est-à-dire que le repreneur associe la ressource réseau reprise à son propre réseau) ; la substitution (le repreneur reconstruit un nouveau réseau : la ressource réseau préalable n'est pas transférée soit parce qu'elle n'a pas de valeur, soit parce que la compétence associée n'est pas transférée).
Ce document est protégé par la loi sur le droit d'auteur. L'utilisation des services d’Érudit (y compris la reproduction) est assujettie à sa politique d'utilisation que vous pouvez consulter en ligne.

https://apropos.erudit.org/fr/usagers/politique-dutilisation/ 


\title{
Le transfert de la ressource réseau lors d'un processus de reprise
}

Sébastien GEINDRE

CERAG, IAE-IUT Valence

MOTS CLÉS

Capital social - Dirigeant - Reprise d'entreprise - Réseaux

\begin{abstract}
L'AUTEUR
SÉBASTIEN GEINDRE est maître de conférences à l'IAE de Grenoble (Université Pierre-MendèsFrance) où il enseigne le management et le management stratégique. II est chercheur au CERAG (Centre d'études et de recherches approfondies en gestion). Ses travaux portent principalement sur les réseaux stratégiques et les reprises d'entreprise. II s'intéresse notamment au rôle du dirigeant dans la construction de la stratégie de la PME. Adresse: IAE-IUT Valence, 51, rue Barthélémy-de-Laffemas, 26000 Valence, France. Courriel : <sebastien. geindre@iut-valence.fr>.
\end{abstract}

\section{RÉSUMÉ}

Le transfert de la ressource réseau est-il possible dans le cadre d'une reprise d'entreprise par une personne physique? Et si oui, comment? Après avoir présenté les concepts de réseau et de réseautage et montré que ceux-ci peuvent être appréhendés respectivement comme une ressource et une compétence de l'organisation et/ou de son dirigeant dans le cadre d'une petite entreprise, nous cherchons à montrer la difficulté de transférer ces actifs dans un processus repreneurial. À partir de quatre cas exploratoires et d'une étude de cas en profondeur, nous proposons d'associer au processus repreneurial une prise en compte spécifique de la ressource réseau en quatre étapes (perception, évaluation, utilisation, valorisation). La prise en main effective de la ressource réseau gérée par le cédant pouvant donner lieu à trois types de valorisation de la ressource: l'appropriation (le repreneur absorbe en l'état la ressource réseau et a la capacité de la mobiliser); l'adjonction (c'est-àdire que le repreneur associe la ressource réseau reprise à son propre réseau); la substitution (le repreneur reconstruit un nouveau réseau: la ressource réseau préalable n'est pas transférée soit parce qu'elle n'a pas de valeur, soit parce que la compétence associée n'est pas transférée). 


\begin{abstract}
This article aims to question the problematic of transferring networks when a small enterprise is taken over (by a person, not by another firm). I present network as a resource and networking as a competence and I show the difficulty of transferring these assets. Thanks to four exploratory cases and one in-depth case study, I underline a four step process to transfer the network resource (perception, evaluation, utilization and valorisation). Then, I identify three ways of handling this resource. (1) Appropriation: the one that takes over absorbs the resource as it was previously and (s)he has the capability to use it properly. (2) Adjunction: the one that takes over associates its own network to the one it has overtaken. (3) Substitution: the one that takes over rebuilds a new network either because it has no value or because (s)he is unable to transfer the competence to handle the network.
\end{abstract}

\title{
RESUMEN
}

¿Es posible transferir el recurso red cuando se vende una empresa ? Y si es posible ¿de qué manera? Tras presentar los conceptos de red y de su optimización como un recurso y una competencia de la empresa y/o del dirigente en el marco de una PyME, hemos intentado demostrar la dificultad de transferir este tipo de activos. Basándonos en cuatro ejemplos exploratorios y un estudio de caso más detenido / detallado/ minucioso, proponemos un proceso en cuatro fases para transferir el recurso red (percepción, evaluación, utilización, valorización) así como tres maneras de apoderarse de este recurso por parte del nuevo dirigente. (1) La apropiación: el comprador de la empresa absorbe la red y tiene la capacidad de utilizarla. (2) La adjunción : el comprador asocia su propia red a la de la empresa comprada. (3) La substitución: el comprador reconstruye una nueva red sea porque la red de la empresa comprada no tiene valor, sea porque el comprador no tiene la competencia de utilizarla.

\section{ZUSAMMENFASSUNG}

Ist es möglich bei der Übernahme durch eine natürliche Person auch ein Netzwerk zu übertragen? Falls ja, wie kann dies bewerkstelligt werden? Nach kurzer Durchsicht der Konzepte zu Netzwerken und Networking, zeigen wir auf, wieso diese als Ressource respektive Kompetenz einer Organisation - oder des Geschäftsführers bei kleinen Unternehmen - aufgefasst werden können und worin die Schwierigkeiten liegen, diese bei der Nachfolge zu übertragen. Auf der Basis von vier explorativen und einer vertieften Fallstudie schlagen wir einen Nachfolgeprozess vor, der die Übertragung des Netzwerkes in vier Schritten berücksichtigt (Wahrnehmung, Bewertung, Nutzung, Verwertung). Die Netzwerkressourcen können auf drei Arten verwertet werden: Aneignung (der Nachfolger übernimmt Netzwerk und die Fähigkeit dieses zu mobilisieren); Hinzufügung (der Nachfolger fügt das neue Netzwerk seinem bestehenden zu); Substitution (ein neues Netzwerk wird aufgebaut, vorheriges Netzwerk wird nicht transferiert, da es keinen Wert für die neue Tätigkeit beinhaltet). 


\section{Introduction}

Les travaux relatifs à la reprise d'entreprise par des personnes physiques (Deschamps, 2002; Picard et Thévenard-Puthod, 2004) ou à la transmission (Cadieux, 2005; St-Cyr et Richer, 2005) montrent la complexité de ces processus. Le risque d'échec des reprises est élevé alors que, paradoxalement, l'enjeu est de taille. Le rapport du cabinet KPMG (2008) sur les risques et opportunités de la transmission des entreprises industrielles réalisé pour le ministère de l'Économie et des Finances français indique que $21 \%$ des entreprises reprises seraient en échec après six ans d'activité tout en évaluant à 700000 les entreprises à reprendre dans les 10 ans à venir. Au Canada, Brouard et Cadieux (2007) indiquent que $41 \%$ des dirigeants de PME prévoient de transmettre leur entreprise avant 2010. Ce chiffre passe à $71 \%$ d'ici à 2015 (FCEI, 2005).

La prise en compte des ressources et des compétences comme facteurs explicatifs de la compétitivité des firmes est aujourd'hui largement établie (Wernerfelt, 1984; Grant, 1991; Hall, 1992; Arrègle, 1995; Teece, Pisano et Shuen, 1997; Tywoniak, 1998). De la maîtrise de ressources et compétences clés dépend directement la capacité des firmes à survivre. Le réseau est une ressource intangible (Hall, 1992) dont le rôle pour l'entrepreneur est déterminant dans la réussite de son entreprise (Baron et Markman, 2003; Davidson et Honig, 2003; Soh, 2003; Walter, Auer et Ritter,2006). Dans le cas spécifique de la PME, a fortiori de la PE, le réseau de l'entreprise est souvent assimilable au réseau du dirigeant (Froehlicher, 1997). Dès lors, comment transférer la ressource réseau de l'entreprise à reprendre?

À partir de quatre cas exploratoires, nous présentons les risques d'échec de reprise liés à une problématique de transfert de la ressource réseau. Nous approfondissons notre question de recherche générale à partir d'une étude de cas en profondeur. Dans un premier temps, nous présentons le cadre conceptuel de cette étude, puis le cadre opératoire et, enfin, nous présentons les résultats et discutons des limites et pistes futures de recherche.

\section{Le cadre conceptuel}

Après avoir présenté le réseau et le capital social comme des ressources, nous les relions aux notions de compétence sociale et de capacité de réseautage afin de mieux comprendre les enjeux du transfert de la ressource réseau dans le cas d'une reprise d'entreprise. 


\subsection{Réseau et capital social : des ressources}

La petite entreprise est un espace ouvert (Marchesnay et Julien,1990) inséré dans un réseau de relations. Le réseau est un système d'acteurs interdépendants, poursuivant un objectif général de coopération. La nature de ces nœuds peut différer: entreprises, hommes, autres organisations. On peut donc a priori différencier des réseaux d'entreprises des réseaux de personnes. Toutefois, comme le montre Granovetter (1985), l'action économique est encastrée dans des structures de relations : un réseau organisationnel est toujours soustendu, conditionné par l'existence d'un réseau de personnes. Dans le cas de petites entreprises, le dirigeant est celui qui va sous-tendre le réseau de son organisation. Plus l'entreprise est petite et plus la confusion entre le réseau du dirigeant et celui de son entreprise sera grande. La qualité du réseau du dirigeant (son capital social) et plus encore sa capacité à structurer et utiliser son réseau (sa compétence sociale) vont impacter la performance de sa firme.

Après avoir longtemps considéré les réseaux comme une forme d'organisation hybride entre marché et hiérarchie (Thorelli, 1986; Powell, 1987), transitoire et instable (Williamson, 1985), celle-ci a finalement été reconnue comme une forme propre distincte du marché et de la hiérarchie (Powell, 1990). La confiance apparaît comme le principal mécanisme de coordination de ces relations (Bradach et Eccles, 1989). Concernant l'allocation de ressources dans les réseaux, «les transactions n'apparaissent pas à travers des échanges discontinus ou par une autorité administrative mais à travers des réseaux d'individus engagés dans des actions mutuelles, réciproques, préférentielles» (Powell, 1990, p. 299). Le réseau favorise l'apprentissage et l'échange d'information. Il crée de l'interdépendance entre les parties, implique des normes de réciprocité et engage la réputation des acteurs. Il représente un modèle viable d'organisation et est constitutif du capital social des individus qui y participent.

La théorie du capital social fait référence à la capacité des acteurs à tirer profit de leurs structures sociales et de leurs réseaux (Bourdieu, 1980; Coleman, 1988). Cette structure relationnelle facilite ainsi certaines actions des individus qui la composent (Coleman, 1988). C'est donc la mise au jour des réseaux du dirigeant qui permet l'opérationnalisation, l'identification et la mesure de son capital social (Davidson et Honig, 2003). D'un point de vue stratégique, le capital social du dirigeant (le potentiel de son réseau) s'apparente à une ressource pour l'entreprise $($ Hall, 1992) qui contribue à la création d'un avantage concurrentiel; il est donc un facteur de performance de l'organisation. C'est un actif stratégique (Wernerfelt, 1984;Arrègle, 1995). Dans le cas de la petite entreprise ou de l'entreprise naissante «le capital social [...] contribue par un effet multiplicateur sur les autres formes de capital 
individuel à différencier les individus quant à leur capacité à innover » (Suire, 2004, p. 127). Il renforce la capacité des individus à percevoir des opportunités (Davidson et Honig, 2003). Il permet l'accès à des formes alternatives de financement (Dussuc et Geindre, 2000) et améliore les performances (C.A., profit, survie à long terme, etc.) dans le cadre d'entrepreneuriat par essaimage (Walter, Auer et Ritter, 2006).

Le réseau est considéré comme une ressource dans la mesure où il offre un mode d'accès privilégié à d'autres ressources et peut ainsi engendrer différentes formes d'avantage concurrentiel durable (Gulati, Nohria et Zaheer, 2000). C'est la maîtrise de relations privilégiées avec d'autres entreprises ou d'autres individus qui permet de bénéficier des ressources des partenaires ou des alliés. Cette maîtrise relationnelle dépend du sentiment de proximité et de confiance existant entre le dirigeant, son entreprise et les parties prenantes. Le réseau est une ressource intangible (Hall,1992) au même titre que la réputation, les brevets, le savoir-faire ou la culture. Elle est donc difficile à observer et à évaluer dans le cas d'une reprise. Il s'agit également d'une ressource dite de position (Hall, 1992). En effet, la relation de réseau ne dépend pas de la volonté ou du comportement de la firme seule et, à ce titre, ne peut pas être contrôlée qu'en interne: elle repose aussi sur le comportement et la volonté des parties prenantes externes. La relation de réseau est donc une ressource qui n'a de valeur que si elle est partagée. Dans le cadre d'une reprise, le transfert du réseau ne dépend donc pas uniquement de la (bonne) volonté du cédant mais aussi de la réaction de l'environnement. Enfin, la ressource réseau est dépendante du facteur humain (Hall, 1992), ce qui l'apparente aux ressources de type savoir-faire ${ }^{1}$. Ainsi, la question de la compétence associée à la maîtrise de cette ressource est critique dans le cadre d'une reprise de PE. En effet, le dirigeant est souvent «le grand architecte» du réseau de sa firme et son capital social part a priori avec lui. Un apprentissage de la pratique du réseau s'avère donc fondamental pour le repreneur.

Le réseau est donc difficile à observer, à évaluer, tributaire de la réaction de l'environnement du cédant et nécessite un apprentissage de son utilisation. Comme le montre Lin (1999), le capital social est encastré dans la structure sociale de l'individu et cette structure est mobilisée en fonction des besoins (objectifs) de l'individu. Si l'on postule que le dirigeant d'une entreprise est l'acteur central,l'administrateur de cette ressource, qu'en est-il au moment de

1. «Quand une compagnie est rachetée, l'acquéreur doit être relativement confiant quant au fait qu'il obtienne les ressources intangibles telles que les brevets mais il ne peut pas être certain qu'il récupérera les ressources intangibles comme le savoir-faire, la culture ou les réseaux qui risquent de "disparaître" » (Hall, 1992, p. 136). 
son départ ? Comment un repreneur peut-il s'approprier son réseau, c'est-àdire d'abord évaluer la structure sociale utile, ensuite s'y connecter, et ce, in fine, pour la mobiliser?

\subsection{Du réseau au réseautage : de la ressource à la compétence}

Grant (1991) nous éclaire sur l'articulation entre ressources et compétences car les ressources ne sont pas productives en soi. Elles nécessitent pour cela «la coordination et la coopération de plusieurs ensembles de ressources. Une compétence est justement la capacité à coordonner ces ressources pour réaliser une activité donnée» (Grant,1991, p. 118). L'articulation entre le concept de réseau (la ressource) et celui de réseautage, c'est-à-dire l'utilisation efficace du réseau (la compétence) peut s'opérer à partir de deux concepts complémentaires: la compétence sociale (Baron et Markman, 2003) et la capacité de réseautage (Walter, Auer et Ritter, 2006).

La compétence sociale se définit comme l'efficacité globale du processus d'interaction avec les autres et, par conséquent, dépend de la capacité à créer et étendre son réseau, à se positionner pour ensuite le mobiliser. Les travaux de Baron et Markman (2003) mettent en évidence trois facteurs structurant le concept de compétence sociale, à savoir:1) la perception sociale, c'est-à-dire la capacité à percevoir justement les autres, leurs caractéristiques, intentions et motivations, 2) la capacité d'adaptation sociale, c'est-à-dire l'aptitude à être à l'aise avec n'importe quel interlocuteur, à entamer une discussion et 3) la persuasion, c'est-à-dire la capacité à faire changer un point de vue ou un comportement chez l'autre ${ }^{2}$. La compétence sociale est donc nécessaire pour construire et augmenter son capital social. Il s'agit d'un savoir-faire individuel. C'est une qualité du dirigeant.

La capacité de réseautage est le second concept susceptible d'articuler la notion de réseau en tant qu'actif de l'entreprise et le réseautage en tant que savoir-faire du dirigeant. Walter, Auer et Ritter (2006, p. 546) la définissent comme l'aptitude de l'entreprise à «initier, maintenir et utiliser des relations avec de nombreux partenaires externes ». La notion de capacité s'entend bien au sens de Teece, Pisano et Shuen (1997), c'est-à-dire comme un savoir organisationnellement intégré et dynamique. En ce sens, la capacité de réseautage se distingue de la compétence sociale dans la mesure où il s'agit d'un savoir de l'organisation et non pas d'une qualité individuelle.

2. Les auteurs proposent des construits théoriques complémentaires mais non validés par l'étude statistique:4) l'impression (capacité à faire bonne impression, à faire passer la bonne impression à l'interlocuteur pour susciter une réaction positive de sa part), 5) l'expressivité (capacité à susciter l'enthousiasme) et 6) l'intelligence émotionnelle (capacité à gérer ses émotions, celles des autres). 
La nature de la compétence associée à la ressource réseau est donc déterminante dans le cadre du processus de reprise de l'organisation. En effet, si cette compétence est essentiellement liée au dirigeant (sa compétence sociale) alors le réseau en tant qu'actif utile, en tant que ressource, n'aura de valeur que si le repreneur possède des qualités relationnelles similaires ou équivalentes. Si la compétence de réseautage est une capacité dynamique de l'organisation (la gestion du réseau s'appuie sur des outils, des routines, des procédures dissociables du dirigeant) alors la ressource est transférable avec l'entreprise.

Si la relation de réseau est considérée comme un actif relationnel, sa construction, son institutionnalisation et son management constituent une compétence. Dans le cadre du processus de reprise, il convient donc de s'interroger sur la nature de cette compétence et notamment d'évaluer si celle-ci est avant tout reliée au dirigeant ou à son organisation.

\subsection{Replacer la ressource réseau dans le processus de reprise d'entreprise}

Le processus de reprise d'entreprise ou processus repreneurial s'articule autour de trois ou quatre étapes clés selon les auteurs. Il diffère du processus de transmission même si certaines phases sont communes et concomitantes. Pour Deschamps (2002), le processus repreneurial s'articule autour de trois étapes: le processus relatif à la prise de décision de reprendre, le processus de reprise à proprement parler et le processus d'entrée dans l'entreprise. Picard et Thévenard-Puthod (2004) relèvent quatre phases: la phase de préparation, l'accord, la transition et le management de la reprise.

Le processus de prise de décision (Deschamps, 2002) est une phase centrée sur le repreneur et son projet de devenir dirigeant: il valide sa motivation, diagnostique ses compétences et entérine le projet de reprendre. C'est l'émergence d'un événement déclencheur qui le pousse à agir et à passer à la deuxième étape. Picard et Thévenard-Puthod (2004) parlent de phase de préparation. Celle-ci étant plus large puisqu'elle englobe la phase de recherche. La césure entre cette phase initiale et la suivante étant la rencontre avec le futur cédant. La ressource étant liée à l'entreprise à reprendre, le processus de prise de décision (ou de préparation) n'est a priori pas critique de ce point de vue.

Pour Deschamps (2002), la recherche est la première étape du processus de reprise qui inclut également l'évaluation, la négociation et la signature de l'accord. Cette phase dite d'accord (rencontres, évaluation, négociation, accord) pour Picard et Thévenard-Puthod (2004) est critique du point de 
vue de la ressource réseau. C'est en effet à ce moment que le repreneur doit prendre en compte l'importance de la ressource réseau et appréhender la nature des compétences nécessaires à sa bonne utilisation. Lors du processus de reprise, l'identification des réseaux clés de l'entreprise, des modalités de leur mise en action et de leurs apports devraient être un élément critique de la phase d'évaluation. C'est pourquoi nous observons si le repreneur potentiel évalue les éléments suivants: quelle(s) structure(s) sociale(s) extérieure(s) à l'entreprise est (sont) mobilisée(s) ? Quelle est la nature de l'encastrement de ces relations (individuelle au niveau du dirigeant ou organisationnelle au niveau de l'entreprise) ? Quel est leur rôle dans la création de l'avantage concurrentiel de l'entreprise?

La dernière phase du processus repreneurial concerne l'entrée dans la structure du nouveau dirigeant. Celle-ci s'opère en deux temps: la transition (le cédant est encore présent et prend part à la passation de pouvoir) et l'investiture (le repreneur devient le pilote de l'entreprise). La phase de transition devrait théoriquement assurer l'intégration du nouveau dirigeant au cœur des réseaux de l'entreprise et la phase d'investiture devrait lui permettre leur prise en main. Nous cherchons à voir comment le repreneur «s'encastre» dans les structures sociales du cédant et ensuite les mobilise.

\section{L'approche méthodologique}

Après avoir justifié le rôle des cas exploratoires, nous présentons le cadre de notre étude de cas principale. Nous expliquons enfin les choix faits en matière de traitement des données.

\subsection{Les cas exploratoires}

L'émergence de notre problématique est fortuite. Lors d'une recherche traitant un autre aspect de la reprise d'entreprise, nous avons été confronté à plusieurs cas de repreneurs en difficulté du fait de la perte de ressources de type réseau, en tout ou en partie. La situation d'échec de ces entreprises nous apparaissait dépendante de cette perte. Parallèlement, la transition réussie d'autres cas indiquait que le transfert réussi de ce type de ressources expliquait, en partie sans doute, le succès des reprises. Nous avons donc opté pour une démarche exploratoire.

La démarche de recherche est donc, dans un premier temps, inductive. Notre objectif étant de faire émerger des éléments susceptibles de préciser les contours de la question de recherche et de l'objet de recherche, nous avons isolé quatre cas pour leur intérêt a priori. Les données ont été recueillies par entretiens semi-directifs centrés. Les thèmes de l'entretien ayant servi à 
élaborer les prémices de notre réflexion traitaient de l'histoire du dirigeant (évaluation de son réseau personnel avant la reprise et positionnement par rapport au réseau du cédant), du déroulement des trois phases du processus repreneurial (prise en compte de la ressource dans l'évaluation de l'entreprise reprise, évaluation puis prise en main et management du réseau de l'entreprise en phase d'entrée), des performances actuelles et de la stratégie mise en place par le repreneur (impact du réseau sur la performance, positionnement des ressources réseau historiques, intégration des nouvelles ressources réseaux, le cas échéant). Pour chaque cas, les repreneurs ont été interrogés en face à face (durée moyenne de l'entretien: deux heures). Un entretien téléphonique confirmatoire a également été réalisé (présentation de l'interprétation du chercheur des situations vécues pour confirmation ou infirmation ou précision). Afin de pallier les limites évidentes du processus de recherche initial (absence de contact avec les cédants, source unique d'information), nous avons prolongé le processus de recherche par une étude de cas unique, longitudinale en observant sur une période de six mois un processus repreneurial.

\subsection{L'étude de cas longitudinale}

Le choix de l'étude de cas se justifie lorsqu'il s'agit d'examiner des phénomènes mal connus ou dans des situations où une nouvelle perspective permet d'enrichir la vision d'un thème déjà exploré (Eisenhardt, 1989). En l'occurrence, le processus repreneurial entre dans cette catégorie mais le transfert de la ressource réseau a été peu observé de manière spécifique. L'étude de cas permet d'appréhender un phénomène complexe, actuel et contextualisé sur lequel le chercheur n'exerce pas ou peu de contrôle (Yin, 1989).

Dans le cadre de cette recherche, il s'agit de présenter un cas unique car il s'agit d'un cas révélateur (Yin, 1989), c'est-à-dire une situation où un chercheur a la possibilité d'étudier un phénomène jusqu'ici inaccessible. Le cas dont nous rendons compte ici est une opportunité d'observation in vivo d'un projet de préparation de reprise d'entreprise entre un dirigeant d'une TPE (quatre salariés) dans le secteur du bâtiment et son successeur potentiel: un étudiant en fin de cycle universitaire nourrissant le projet de reprendre cette entreprise. L'objectif initial étant double: observer le phénomène du transfert de la ressource réseau (rapidement identifié comme un élément clé de la réussite de transfert) et enclencher le processus de reprise. Il s'agit aussi, à partir de cette étude de cas en profondeur, d'élaborer de nouvelles hypothèses. Le mode de raisonnement se veut donc abductif ${ }^{3}$ dans la mesure

3. «En management [...], il est fréquent que le chercheur explore un contexte complexe, emprunt d'observations nombreuses de différentes natures et au premier 
où nous avons multiplié les rencontres avec le repreneur potentiel lors de son immersion pour lui présenter les connaissances théoriques liées aux fonctionnements des réseaux, à leur construction et à leur observation (méthodologie) afin de les confronter à la situation (terrain).

\subsection{Le traitement des données}

Pour le traitement des données, nous avons suivi le processus de la grounded theory (Glaser et Strauss, 1967; Glaser, 1978). Cette démarche suit un processus en trois points principaux. Il convient d'abord de produire des données. Glaser (1978) privilégie ce terme à celui de collecter dans la mesure où le chercheur est actif dans ce processus et agit sur les données (elles n'existent pas à l'état naturel). Ensuite, il incombe au chercheur de traiter ces données afin de passer d'un niveau factuel à un niveau conceptuel. Cette étape inclut les actions de codage ouvert (codage large) et de codage sélectif (sélection des catégories clés). Le codage est le processus de passage des données factuelles aux catégories. Une catégorie est issue des faits, mais n'est pas un fait, c'est un élément de théorie, un concept. Enfin, le chercheur produit un résultat, c'est-à-dire organise ces informations, les présente et les diffuse (tri et rédaction). Les codages retenus apparaissent dans les tableaux de synthèse ( $c f$. entrées des tableaux 1 et 2 ).

\section{Discussion des résultats}

Après avoir décrit les différents cas et présentés les résultats, ceux-ci sont discutés.

\subsection{Phase exploratoire}

Cas 1. À la suite de son divorce, madame Cahun, 49 ans, souhaite retrouver une activité professionnelle pour s'assurer un revenu (elle était totalement dépendante de son ex-mari) et diriger son affaire. Bénéficiant d'un capital de départ, elle fait le choix de reprendre un commerce de vêtements dans le centre de Grenoble (agglomération de 500000 habitants environ). En quelques mois, le commerce à reprendre est identifié (l'entremetteur est une agence immobilière). Sa connaissance du secteur en tant que consomma-

abord ambiguës. Il va tenter alors de structurer son système d'observations pour produire du sens. En sciences sociales, l'objectif n'est pas réellement de produire des lois universelles mais plutôt de proposer de nouvelles conceptualisations théoriques valides et robustes, rigoureusement élaborées. On dit alors que le chercheur procède par abduction (Eco, 1990) ou adduction (Blaug, 1982)» (Charreire et Durrieux, 1999, p. 60). 
trice et son histoire personnelle (elle est fille de commerçant) lui suffisent à valider son choix. Rapidement, deux problèmes inattendus au moment de la reprise apparaissent: la clientèle se détourne, bien que l'offre ait peu changé (au départ, il y a continuité de la stratégie marketing). Ensuite, au moment de faire évoluer l'offre, elle est incapable de mobiliser le réseau de fournisseurs de l'entreprise tout en éprouvant de grandes difficultés à fidéliser les nouvelles clientes.

Dans sa phase d'évaluation, madame Cahun, consciente du risque pris par un transfert de propriété dans un commerce, a cherché à sécuriser sa démarche, d'abord en vérifiant l'existence d'un fichier commercial des clientes. De même, elle s'était entendue avec la cédante sur une période de transition afin de prendre en main les relations fournisseurs. Lors de la phase d'entrée, la cédante se retire rapidement (quatre jours) au bénéfice d'une clause mal négociée (l'accompagnement était prévu pour une durée d'un mois sauf «en cas de contrainte indépendante de la volonté de la cédante»). Rapidement, le chiffre d'affaires décline. Soucieuse de mobiliser son réseau de clientes, elle active le fichier pour une opération commerciale et constate qu'il n'a pas été actualisé, sa valeur réelle est faible. De plus, les clientes historiques ne retrouvent pas la relation de confiance qu'elles avaient avec la cédante. Elles attendaient une offre globale: vêtement plus conseils de type relooking, or, cela n'est pas la compétence de madame Cahun qui voyait son rôle limité à la vente de vêtements. Le chiffre d'affaires chute de $50 \%$ en quelques semaines. Devant cette situation, madame Cahun souhaite entreprendre un repositionnement complet afin de trouver une clientèle de substitution. Elle souhaite se tourner vers les fournisseurs habituels du magasin mais, faute de fichier, elle doit reprendre toute la facturation pour trouver leurs coordonnées. Elle découvre le fonctionnement des salons du prêt-à-porter, se trouve confrontée à des interlocuteurs parlant anglais, langue qu'elle ne maîtrise pas... Madame Cahun réussit à modifier son offre. $\mathrm{La}$ nouvelle clientèle représente deux tiers de son chiffre d'affaires. Elle reste volatile (incapacité à nouer des relations fortes de type réseau, on reste dans des relations de type marché). Sa situation est précaire.

Le cas illustre les problèmes d'évaluation de la ressource réseau et de la compétence associée. Dans ce cas, les ressources ont été identifiées mais pas les compétences relationnelles qui allaient avec et qui dépendaient directement de la cédante (le savoir-faire en termes de conseil structurait la relation de confiance que la cédante avait avec ses clientes). En l'absence de ce savoir-faire, les relations sont de type marché standard et la ressource réseau n'en est plus une. Il en est de même pour les relations fournisseurs. Madame Cahun fondait son repositionnement marketing sur le postulat que les fournisseurs sont connus et facilement accessibles (capacité de réseautage). Or, 
en l'absence de fichier fiable (absence de mémoire organisationnelle) et surtout de transfert de procédure (prévue initialement lors de la transition), la compétence relationnelle n'a pas non plus été transférée. Devant l'impossibilité de mobiliser les ressources réseaux de l'entreprise, madame Cahun doit reconstruire un tissu relationnel.

Cas 2. Monsieur Cadeux est un homme de 53 ans, ingénieur process dans le secteur mécanique. Après avoir exercé des fonctions de direction dans des grands groupes au niveau de sites productifs, il décide, à la suite d'un bilan de compétences et de la mise en place d'un plan de départ volontaire, de relever un dernier défi professionnel en reprenant une structure. La cible est une TPE de cinq salariés réalisant un million d'euros de chiffre d'affaires. Elle est spécialisée dans l'emboutissage pour la sous-traitance automobile et travaille pour un client unique (l'entreprise a été créée par essaimage de l'atelier d'emboutissage du donneur d'ordres). Il s'agit d'une situation de quasi-intégration, mais la TPE bénéficie d'une souplesse que n'avait pas l'atelier lorsqu'il était préalablement intégré ainsi que d'une capacité de stockage qui fait défaut au donneur d'ordres. Lors du processus de reprise, monsieur Cadeux relève trois relations clés. Premièrement, la relation avec le client est évidemment indissociable de l'existence même de l'entreprise (un contrat de partenariat avec une garantie de six mois de chiffre d'affaires lie l'entreprise et son donneur d'ordres). Deuxièmement, la relation avec le cédant est également clé. En effet, lors du plan de reprise, monsieur Cadeux a clairement prévu de diversifier sa base client et a besoin pour ce faire des compétences techniques du cédant en matière de conception (le cédant développe, à la suite de la cession de son entreprise, un bureau d'études dont le premier client sera son ancienne entreprise); de la réussite de la reprise dépend aussi la réussite du projet de création du cédant. Troisièmement, la relation avec le banquier est également importante compte tenu de la situation particulière et risquée (client unique) de l'entreprise. C'est par ailleurs le banquier qui a mis en relation le cédant et le repreneur; le banquier du cédant le suit avec confiance depuis la création de l'entreprise.

Le réseau, tel qu'il est, est clairement source de danger. Cet élément-là n'échappe évidemment à personne (repreneur, cédant et banquier). La réussite de la reprise dépend donc de la capacité du repreneur à élargir son activité. Or, le banquier n'a pas souhaité financer l'achat de l'entreprise bien qu'il eût servi d'entremetteur. Le chargé d'affaires a changé d'agence et son remplaçant, n'ayant pas vécu l'historique de la relation avec le cédant, estime qu'il est trop dangereux de financer la reprise (d'une relation encastrée socialement entre le banquier et le cédant, on bascule vers une relation de marché entre le nouveau banquier et le repreneur, et, dans ce cadre, la relation est trop risquée). De plus, quelques mois après la reprise, le donneur d'ordres 
dénonce son contrat de partenariat sans pour autant mettre un terme à la relation. Le repreneur affirme qu'il s'y attendait mais que le client a toujours besoin de lui.

D'une manière générale, le risque représenté par le transfert de la ressource réseau a été largement pris en compte. Il a donné lieu à évaluation et monsieur Cadeux a élaboré son plan de reprise en fonction. Ainsi, il a négocié une phase de transition de deux ans (six mois intensifs, six mois à temps partiel et un an à la demande) avec le cédant et en intégrant le rôle joué par l'épouse de celui-ci (comptable). Le déroulement est le suivant: en janvier 2006, monsieur Cadeux apprend l'existence de l'entreprise à reprendre, en février, il prend contact avec le cédant et signe le compromis en mai. Fin mai, le repreneur signe un contrat de partenariat entre la société qu'il a créée pour la circonstance et l'entreprise cible. En juillet, il devient DG de l'entreprise et la société devient holding. L'épouse de l'ancien dirigeant devient PDG jusqu'en juillet 2007, date à laquelle monsieur Cadeux devient PDG et l'épouse de l'ancien dirigeant retrouve une fonction administrative (gestion comptable). Son départ est finalisé en mai 2008. Pour la remplacer, une étudiante en formation en alternance est recrutée dès 2007. L'objectif de cette intégration progressive est d'abord de se focaliser sur le technique la première année afin de construire une relation équilibrée et fiable avec le cédant (et son bureau d'études); ensuite, le maintien de l'épouse du dirigeant permet d'assurer la continuité avec le client tout en se garantissant un lien solide avec le bureau d'études de son mari. Enfin, à l'issue de cette période, le repreneur espère avoir suffisamment avancé en termes de développement de nouveau(x) marché(s) pour limiter le poids du client historique et avoir reconquis la confiance du banquier historique afin qu'il rachète ses crédits contractés auprès de deux autres banques pour l'achat de l'entreprise mais qu'il juge trop chers.

Ce cas souligne le caractère clé de la phase d'évaluation où s'élabore la stratégie de redéploiement du réseau. Monsieur Cadeux avait parfaitement évalué les enjeux du réseau (il avait renoncé à une autre opportunité de rachat au préalable car il avait jugé intransférable la ressource réseau $\left.{ }^{4}\right)$. Toutefois, la mise en place d'un nouveau réseau efficace ou la sortie d'une relation réseau trop engageante prend du temps en l'absence de réseau du repreneur.

Cas 3. Monsieur Catrois a 44 ans. Il a travaillé dans le secteur du bâtiment (plombier) depuis le début des années 1980 et a été artisan de 1997 à 1999.

4. «Avant de rependre X,j'avais repéré un bureau d'études. Le problème, c'est que la ressource clé était le dirigeant lui-même et notamment son réseau personnel. Or, c'était injouable. [...] le mieux placé pour reprendre était son ingénieur calcul. Du coup, j'ai préféré laisser tomber les activités de négoce et les bureaux d'études.» 
À la suite d'une opportunité (décrite comme telle), il reprend une activité salariée jusqu'en 2007 et devient chef de chantier (il est mieux payé, gère des chantiers importants et supervise une quinzaine de personnes). Après avoir souhaité changer d'employeur, il a eu connaissance de la cession d'une entreprise de dépannage et rénovation en plomberie (qu'il connaissait) plutôt spécialisée dans la clientèle professionnelle (syndic, régie d'immeuble) et s'est laissé tenter par le challenge avec comme objectif de moderniser l'affaire. Il a été conforté dans sa décision par trois autres chefs d'entreprise du secteur qu'il connaissait et qui ont investi dans l'affaire. La reprise ne se serait pas faite sans l'appui moral des trois associés et leur appui financier (le repreneur détient $30 \%$ du capital, les deux associés ont $25 \%$ et un quatrième a $20 \%$; leurs apports ont été financés par emprunt auprès des banques des associés). L'entreprise de neuf salariés réalisait un chiffre d'affaires de $900000 €$ avant la reprise sans évolution notable depuis plusieurs années. Dès la reprise, l'entreprise a été immédiatement réorganisée en interne avec la promotion d'un (jeune) salarié au poste d'adjoint du dirigeant afin de piloter l'opérationnel et la gestion des chantiers. La promotion a été négociée en amont de la signature de la vente. Deux personnes ont été recrutées par la suite et le chiffre d'affaires a évolué en proportion. Aucun client n'a été perdu et de nouveaux clients ont été démarchés. Le dirigeant essaie de se concentrer sur des dimensions stratégiques. Il souhaite entrer sur le marché des particuliers et mettre à profit ses réseaux pour les marchés de la construction neuve.

Dans ce cas, la ressource réseau est avant tout composée des interlocuteurs du marché des professionnels de l'immobilier (les clients de l'entreprise). Ce réseau ne semble pas reposer sur le capital social de l'ancien dirigeant mais plus sur la capacité de réseautage de l'organisation (les interlocuteurs des régies connaissent les dépanneurs, par exemple). De plus, du fait de son parcours professionnel, monsieur Catrois connaît un grand nombre de ces interlocuteurs. Le transfert de cette ressource n'a posé aucun problème. Les relations amont (avec les fournisseurs) sont de type marché (absence d'encastrement), ce qui facilite le transfert. La principale difficulté de monsieur Catrois concerne sa capacité à associer son propre réseau, potentiellement apporteur d'affaires pour l'entreprise, au réseau de l'entreprise.

Cas 4. Monsieur Caquatre est un ancien cadre d'une grande entreprise de composants électriques (responsable d'un service après-vente gérant 160 personnes). Il a également dirigé l'export chez un grand fabricant d'articles de sport. Il est technicien de formation puis ingénieur (formation continue) et titulaire d'un MBA (formation continue). L'entreprise reprise est une TPE de quatre salariés réalisant un chiffre d'affaires de un million d'euros en 2006. L'entreprise réalise du commerce de gros dans l'électricité, sur quatre familles de produits techniques, avec des licences nationales exclusives pour deux d'entre eux et régionales pour les deux autres. 
Dans ce cas, les relations avec les fournisseurs de l'entreprise sont ancrées organisationnellement. Leur transfert est aisé. Au niveau aval, le cédant avait des relations historiques avec ses clients. Le repreneur, issu du même secteur industriel, possède également de solides connexions, notamment avec son ancien employeur, client de l'entreprise reprise, ainsi qu'avec d'autres interlocuteurs du secteur. Toutefois, afin de garantir la transition, tous les clients de la structure ont été vus en binôme. L'appropriation du réseau d'affaires de l'entreprise a donc été facile, immédiate et ne s'est traduite par aucune perte de clientèle ou remise en cause de partenariat. Chaque contact avec les clients a notamment été l'occasion pour le repreneur d'envisager un élargissement de la relation lorsque cela était possible. Deux cas de figures sont apparus: 1) les relations bloquées sur l'existant et 2) les relations porteuses. Dans le premier cas, l'entreprise réalise un chiffre d'affaires plafond avec le client qui ne manifeste pas le besoin d'augmenter ses flux ou d'ouvrir de nouvelles bases d'échange (élargissement de l'offre). Dans le second cas, l'entrepreneur souhaite mobiliser la relation existante pour faire évoluer son offre du négoce pur vers le découpage à façon. Il s'agit de faire évoluer des relations de type liens faibles vers des relations plus structurées, plus interdépendantes en apportant un service nouveau, «sur-mesure», créateur de valeur pour les deux parties. Cette transformation suppose un engagement plus fort (conception et développement de pièces spécifiques). Par ailleurs, le repreneur arrive avec son propre réseau qu'il souhaite adjoindre à celui de l'entreprise (réseau de contacts professionnels à l'étranger dans une perspective de développement international).

L'analyse de ces cas avait pour but de prendre en compte la ressource réseau dans le cadre du processus repreneurial (tableau 1 ). Après avoir identifié avec les repreneurs les relations de réseau clés de l'entreprise et compris comment cette ressource a été transférée lors du processus de reprise, nous nous sommes intéressé à la stratégie mise en place par les repreneurs lors de la prise de contrôle de leur entreprise.

Les quatre cas mettent en évidence l'importance de bien percevoir le rôle clé joué par les réseaux. Ce point a été ignoré dans le cas 1 . Si les autres repreneurs semblent avoir été plus vigilants, le cas 2 met en évidence la problématique de l'évaluation de ces relations (la relation avec la banque était tributaire du chargé de clientèle). Les cas 3 et 4 montrent qu'une évaluation satisfaisante des réseaux facilite la reprise de cette ressource, et ce, a fortiori, si la compétence (capacité de réseautage) est associée au transfert de la ressource. Le cas Immo va nous permettre d'approfondir ces deux constats. Enfin, les cas préparatoires mettent au jour plusieurs stratégies d'utilisation de la ressource réseau (substitution, appropriation, adjonction). Ces stratégies n'étant pas toujours totalement délibérées. 


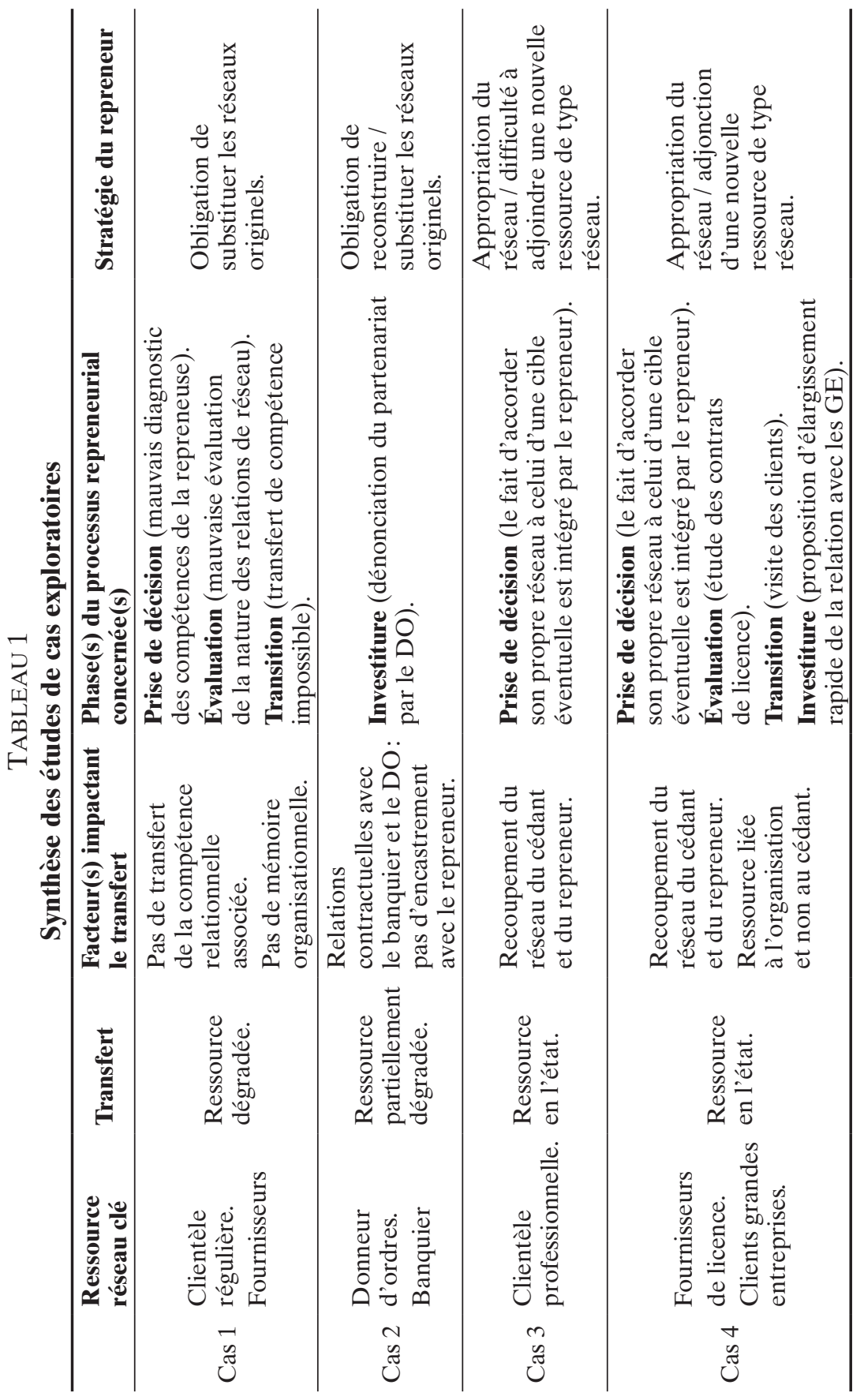




\subsection{Le cas Immo}

La société Immo est une TPE de quatre salariés exerçant principalement l'activité de lotisseur aménageur. Ponctuellement, elle réalise de la réhabilitation de bâtiments. Elle a à son actif la réalisation de plusieurs transformations de châteaux ou d'anciens hôtels en logements ainsi que la réalisation de près d'une dizaine de lotissements dans la région grenobloise. Cette société réalise un chiffre d'affaires moyen de deux millions d'euros chaque année avec un résultat oscillant autour de 200000 euros par an. Toutefois, du fait de problèmes de santé du dirigeant, du désengagement progressif des associés (départ en retraite programmé, cessation progressive d'activité en cours), la société risque de cesser son activité. L'observation a porté sur l'immersion d'un étudiant en fin de cycle universitaire pendant une durée de six mois (avril à septembre 2008). Son objectif était de valider ou d'invalider la possibilité de reprendre cette entreprise. Il n'y a aucun lien de parenté entre le cédant et le repreneur potentiel. Toutefois, la relation est socialement encastrée:l'étudiant a réalisé un stage préalable dans la structure en 2006, les deux protagonistes se connaissent et le projet de reprise a été envisagé à ce moment. Le dirigeant et le père de l'étudiant sont amis. Enfin, le dirigeant n'a pas d'enfant.

Nous retranscrivons les éléments du cas Immo en trois points : d'abord, en replaçant les protagonistes et leur projet de reprise par rapport au processus repreneurial. Ensuite, nous présentons les éléments relatifs à la ressource réseau de ce cas et, enfin, les éléments liés à la compétence relationnelle de son dirigeant.

\subsubsection{Le processus repreneurial de l'entreprise Immo}

Le processus repreneurial tel qu'il s'est déroulé dans ce cas ne recoupe pas complètement les processus standards évoqués dans la littérature. Nous notons trois différences.

D'abord, la phase de prise de décision n'est pas totalement validée: le repreneur potentiel est là pour tester son projet et se tester. La reprise d'une entreprise n'est pas une finalité professionnelle certaine. En effet, le repreneur potentiel est conscient d'être animé d'envies contradictoires: diriger, bénéficier d'un statut social qu'il valorise (dirigeant de société), avoir un niveau de vie confortable (éléments correspondants à la situation actuelle du dirigeant), ce qui fait de l'entreprise Immo une opportunité; mais, en même temps, il est curieux du monde qui l'entoure, avide de le comprendre et d'avoir des activités de réflexion et craint que l'activité de direction de l'entreprise Immo ne le prive de cette possibilité de «prendre le temps de réfléchir ». Il conçoit donc l'entreprise Immo comme une opportunité unique 
dans le sens où son projet de reprise n'a de sens que pour cette entreprise (il exclut de se lancer dans un autre processus repreneurial). La fin de son cycle d'études supérieures peut être ici appréhendée comme un élément déclencheur possible. Âgé de 27 ans, il reconnaît que la pression sociale (famille, amis) l'incite à «trouver un vrai métier».

Ensuite, la réflexion autour de ce projet de reprise est amorcée depuis son premier stage et a en partie conditionné son choix d'études entre les deux périodes (formation en management et entrepreneuriat). Le repreneur a également pour objectif d'évaluer la faisabilité du transfert de direction. Conscient du rôle clé de la ressource stratégique que représente le réseau du dirigeant, la période d'immersion doit servir à évaluer le réseau et à commencer à se l'approprier (phase d'évaluation relative au processus de reprise).

«Sans réseau, dans ce métier tu n'es rien.»

«Si l'on ne connaît personne, qu'on ne développe pas son réseau, on ne peut rien faire. Absolument rien. Sans ce réseau, je ne pourrais pas travailler et les autres m'empêcherait de travailler.» (Le dirigeant, quand on l'interroge sur ses dépenses.)

[Le cédant, face au repreneur]

«Envisager de reprendre cette entreprise sans engager une réflexion sur le "réseau" de son dirigeant serait plus qu'une erreur. Celui-ci semble en effet être, plus que tout autre chose, la grande force de cette entreprise, sa principale ressource ou, plus exactement, son mode préférentiel d'accès aux ressources (information, compétences, flux financiers, etc.).»

«Si la performance de cette entreprise semble bonne, il est impensable de la maintenir ou de l'augmenter sans ce réseau ou, tout du moins, sans une partie de ce réseau.»

[Le repreneur]

Enfin, la présence du repreneur au côté du cédant de manière intensive correspond généralement à la phase de transition. Or, ce n'est clairement pas le cas puisqu'il n'y pas eu d'accord. Une proposition de collaboration en vue d'une cession à terme a certes été discutée, mais l'entreprise n'a pas fait l'objet d'une évaluation comptable et aucun accord, même tacite, n'est passé entre les parties. Notons que la décision de reprendre n'est pas uniquement du ressort du repreneur (si celui-ci valide son projet et conclut à la faisabilité 
du transfert des ressources réseaux). En effet, le cédant potentiel est dans une situation paradoxale: à la fois malade, âgé de 60 ans, financièrement à l'abri et face à une opportunité de cession, il demeure très attaché à son entreprise car, célibataire et sans enfant, ses réseaux professionnel et personnel se confondent (membres de la famille/associés, amis/relations d'affaires).

«Grâce à la coopération du dirigeant, nous avons pu l'accompagner dans la majeure partie de ses activités personnelles qui, en fait, sont pratiquement indissociables de ses activités professionnelles. D'ailleurs, le dirigeant reçoit très souvent ses contacts professionnels chez lui pour des réunions mais aussi lors de dîners où se mélangent le professionnel et le non-professionnel, sans qu'un but précis soit défini.»

[Le repreneur]

Le transfert éventuel n'a pas été planifié et si on devait replacer la situation du dirigeant d'Immo par rapport au processus de désengagement de Cadieux (2005), celui-ci se situerait au début de la phase 3 (préparation, intégration apprentissage, durée moyenne deux à sept ans).

\subsubsection{L'évaluation de la ressource réseau}

Conscient de l'importance de la ressource réseau, le repreneur a donc, dans un premier temps, observé le mode de fonctionnement du dirigeant en l'assistant puis en prenant à sa charge certaines activités courantes (prospection, contact, montage de projets, etc.).

«Pendant six mois, la mission a été d'assister le dirigeant dans son travail quotidien. Les journées consistent, en apparence, en un travail permanent de prospection (aller voir des terrains, des bâtiments) et d'administration (rendez-vous avec les notaires, les acheteurs, les vendeurs, les bureaux d'études, les géomètres, etc.).»

«Ce dirigeant travaille essentiellement de chez lui. En fait, ces bureaux sont vides, il n'y passe que rarement, le téléphone est même coupé. On pourrait pratiquement croire cette entreprise en faillite ou, tout du moins, en sommeil. Ce serait sans compter les appels ininterrompus, 
sur son téléphone portable: amis, notaires, élus, agents immobilier, promoteurs, architectes, directeur de services techniques, etc. C'est une valse continuelle de coups de fil, mêlant affaires personnelles et professionnelles, les “tu”, les “vous", les “nous” le sérieux et l'humour.»

[Le repreneur]

Rapidement, il a fallu rendre matériel le tissu relationnel du dirigeant. En effet, comme nous l'avons évoqué dans les cas exploratoires, l'absence de mémoire organisationnelle rend plus difficile le transfert de la ressource réseau. En l'absence de quelconques fichiers, il s'est avéré nécessaire de créer cette mémoire. La démarche retenue fut de mobiliser l'agenda du dirigeant afin de relever les nœuds de son réseau. En pratique, l'agenda ne retenait que le formel; or, il est apparu que les relations les plus riches étaient plutôt informelles et souvent inopinées. Chaque contact a donc été répertorié pendant toute la période commune (à partir du deuxième mois) et son rôle précisé. Cette observation a fait émerger deux types de réseaux: un réseau dit technique, qui regroupe les compétences nécessaires à l'élaboration du produit fini (géomètre, entreprise de BTP, bureau d'études pour la voirie et les réseaux divers ou VRD). Partenaires historiques d'Immo, ces entreprises indépendantes collaborent systématiquement ensemble sans qu'Immo recoure au marché pour négocier des conditions différentes avec des concurrents éventuels. Par ailleurs, ces entreprises travaillent pour d'autres donneurs d'ordres. Il n'existe aucun contrat entre ces entreprises. Seules la confiance, l'habitude, l'amitié et l'estime entre les dirigeants les lient. Ce réseau a facilement été identifié.

Le réseau le plus complexe à appréhender est constitué de la multitude de relations informelles qui alimentent en information le processus productif (essentiellement prospectif) du dirigeant. Ces relations sont nombreuses, hétérogènes (diversité des acteurs rencontrés), isolées (les contacts sont peu redondants entre eux), orchestrées par le biais de canaux divers, planifiées ou, au contraire, totalement fortuites.

Dès la première semaine, nous avons rencontré plus de 22 personnes différentes en cinq jours avec lesquels nous avons eu plus d'un contact, un entretien d'au moins cinq minutes à l'occasion de rendez-vous, de réunions, de petits-déjeuners, cafés, déjeuners, apéritifs, dîners, etc. 
Vingt-deux contacts peu redondants (les personnes ne se connaissent pas ou peu entre elles), tous apporteurs d'éléments nécessaires au fonctionnement de l'activité.

Afin d'offrir une vision claire du réseau, nous avons listé dans l'ordre d'apparition les contacts que le dirigeant a eus et bien souvent créés. (...) [pour] les mois de mai et de juin 2008, 45 relations ont été identifiées.

[Le repreneur]

Afin de rendre intelligible et observable le réseau, il a fallu répertorier les nœuds mais aussi certaines de leurs caractéristiques que le dirigeant avait l'habitude de prendre en compte. Ainsi, de manière systématique, il s'intéresse à l'activité professionnelle de la personne, à celle de son conjoint, à son patrimoine, à son milieu social et, lorsque cela est possible, à un centre d'intérêt. De plus, pour chaque nœud, le repreneur a pris en compte l'antériorité de la relation (sur la période concernée, 20 contacts sont nouveaux [44,5\%], l'antériorité moyenne des autres relations est de 10 ans environ). On recense parmi l'ensemble de ces contacts des artisans (11), des professionnels de l'immobilier (8), des politiques (4), mais surtout des apporteurs d'information potentiels qui, du fait de leur position (élu ou autre forme d'ancrage territorial), jouissent potentiellement d'un accès direct à une information rare ou précoce (notaires, ouvriers des services techniques d'une ville ou d'un village, cafetiers, restaurateurs, médecins, policiers municipaux, soit 17 personnes) ou indirect, à travers l'activité du conjoint (p. ex., notaire, maire, élu) ou la situation de la famille (milieu agricole - quatre cas - ou aristocratique - deux cas). Les informations prisées sont essentiellement liées à la mise en vente de terrains constructibles (rôle clé des propriétaires terriens), à la modification de plan d'occupation des sols ou aux lancements de projets immobiliers (rôles des élus).

En définitive, la structure, la composition du réseau n'est pas étonnante et pourrait être agencée à distance avec un annuaire de professionnels, les listes des élus locaux et les plans d'occupation des sols des communes (toutes ses informations étant publiques). Ce qui en fait sa valeur, c'est la capacité du dirigeant à se positionner auprès de ses interlocuteurs, soit pour avoir accès à une information avant le marché, soit pour se faire reconnaître comme un offreur à part (digne de confiance, donc appartenant au réseau) face à une demande. C'est donc, dans ce cas, moins le réseau qu'il faut obtenir (la 
ressource) que la pratique du réseautage, c'est-à-dire la capacité à se l'approprier, à le gérer (la compétence). Cet élément n'ayant pas échappé au repreneur dans son évaluation de la ressource:

En définitive, le principal savoir-faire de ce dirigeant n'est pas technique, il est managérial: il utilise son réseau pour agréger de l'information et des compétences de manière souple et adaptable: il répond ainsi [...] aux besoins du marché. Son réseau et sa propre capacité à l'animer sont les facteurs clés de succès de son entreprise, les leviers majeurs de sa performance.

[Le repreneur]

\subsubsection{La compétence de réseautage}

Au-delà de l'identification des liens du réseau, c'est le processus de construction et d'animation de ces liens qui retient l'attention. Cette compétence semble se fonder sur les éléments suivants: la capacité à accéder à des personnes très différentes (statut et origine sociale, patrimoine), à susciter leur confiance et à les relier à son propre univers.

[Le] dirigeant a une démarche consciente de construction du réseau qui vise à tisser des liens de qualité avec le plus grand nombre de gens possible. [...] Il a, selon ses propres mots, une démarche d'orpailleur, il recherche des opportunités et surtout, il croit dans «la richesse qu'il y a dans les gens ».

C'est bien simple, grâce à une mémoire assez exceptionnelle [...] il fait souvent référence à des détails vieux de dizaines d'années, il connaît «tout le monde» ou, tout du moins, trouve toujours un lien même éloigné avec les personnes qu'il rencontre dans le but de les sécuriser, de leur donner confiance, de se positionner (il fait du «name-dropping») ou tout simplement de les surprendre. C'est une démarche consciente et volontaire.

[Le repreneur] 
En outre, la valeur de ces personnes est liée aux propres ressources qu'ils sont susceptibles d'apporter ainsi qu'à leur propre capacité à tisser des relations au-delà du giron du dirigeant.

Il s'agit pour le dirigeant de trouver un contact qui va lui permettre de réaliser à court terme une affaire ou de débloquer une situation. [...] Pour réaliser un lotissement important et surclasser le groupe immobilier concurrent aux moyens financiers plus importants, [le dirigeant] a pioché dans ses connaissances [...] X est ressortie comme la personne la plus implantée sur la région. Le dirigeant lui a exposé le problème, celui-ci a réfléchi aux personnes qu'il connaissait et l'a mis en relation avec Y, lui-même fortement implanté et intéressé par le projet, qui a contacté $\mathrm{Z}$, architecte, qui l'a mis en contact avec le maire de la commune pour connaître son point de vue sur le développement de sa commune. [Il crée] un sous-réseau dédié à une affaire précise et configuré sur mesure de telle sorte que le niveau d'information et de compréhension des enjeux locaux soit supérieur aux groupes immobiliers dont la démarche est plus administrative. Cette technique permet à moindres frais de bénéficier d'un niveau d'information de qualité et d'être extrêmement réactif et adaptable aux évolutions de la négociation.

Cette capacité qu'ont les gens [...] à créer des liens peut être résumée, en cas de problème du dirigeant qui a épuisé ses propres contacts, par leur capacité à répondre à la question « tu ne connaîtrais pas quelqu'un qui..?». Nous avons à plusieurs reprises pu tester ce mécanisme pour des affaires plus ou moins importantes... et avec succès! En définitive nous avons donc réalisé un tableau qui reprend les deux critères «classiques» et de «liens purs». Ce qui nous a permis de nous rapprocher de ce que nous ressentions en termes d'importance relative des différentes personnes sur le terrain.

[Le repreneur]

Ces différentes observations montrent qu'on retrouve les qualités évoquées par Baron et Markman (2003), à savoir les éléments de la compétence sociale. En outre, cette capacité à articuler de l'information en provenance de mondes différents illustre parfaitement la maîtrise de trous structuraux (Burt, 1995) et traduit un capital social élevé. 


\subsection{Discussion des résultats}

$\mathrm{Au}$ final, que nous apprennent les échecs ou succès relatifs des cas exploratoires en matière de transfert de la ressource réseau? Que pouvons-nous tirer de la difficulté de la reprise de l'entreprise Immo? L'apport de ces cas est discuté en lien avec tout le processus repreneurial.

\subsubsection{La prise en compte de la ressource réseau en amont de la reprise}

La prudence des repreneurs 2, 3 et 4 montrent que la prise de conscience de la ressource réseau doit exister en amont de l'évaluation. Elle contraste avec la naïveté du cas 1 . Ainsi, contrairement à l'avis émis dans la section 1.3 sur la dimension non critique de la ressource réseau à ce moment du processus repreneurial, nous postulons que le repreneur doit être conscient (donc informé, sensibilisé, à défaut d'en avoir fait l'expérience) du rôle clé joué par les réseaux pour ensuite être capable de matérialiser cette ressource. Les cas exploratoires soulignent de manière assez attendue que les relations avec les clients et les fournisseurs, les banquiers, ont une importance cruciale pour la réussite de la reprise. Toutefois, le caractère réticulaire de celles-ci n'a pas toujours été appréhendé. La perception du rôle économique de la relation est bonne mais pas celle du rôle clé que joue la confiance dans le processus de création de valeur.

Ainsi, dans le cas 1, les clientes sont perçues uniquement comme des acteurs économiques satisfaisant un besoin d'achat de vêtement. Or, dans la pratique, pour une part significative des clientes (les plus régulières), cet achat est conditionné par la confiance qu'elles entretiennent avec la cédante et par sa capacité à leur fournir un produit associé un conseil de confiance. Cette dimension-là n'a pas été appréhendée par la repreneuse qui envisage le commerce de vêtement comme de la mise à disposition d'articles, alors qu'il s'agit en fait d'aider au choix, et ce, de manière crédible. On retrouve cette posture dans le cas 2 où la relation avec la banque est dénoncée sitôt que le rapport de confiance est rompu du fait de l'arrivée du nouveau chargé d'affaires (le rapport avec le chargé ne débouche pas que sur une mise à disposition de financement, mais inclut un degré de confiance qui permet une prise de risque plus grande). Si le départ du chargé était imprévu, le risque lié à cette relation n'a pas été correctement mesuré et s'est traduit par un rallongement du temps de recherche de crédit et l'augmentation de son coût. À l'inverse, dans les cas 3 et 4, les relations de confiance ont été maintenues du fait de la compétence du repreneur, de sa connaissance préalable des clients (cas 3) ou de sa démarche de mise en relation faite conjointement avec le cédant (cas 4). Dans les deux cas, les repreneurs bénéficiaient d'une connaissance des pratiques du secteur et avaient conscience du caractère 
primordial d'instaurer ou de maintenir un niveau élevé de confiance avec le réseau. Ce dernier point souligne l'effort à faire du repreneur pour dialoguer avec les partenaires de l'entreprise, faire montre de ses compétences et aptitudes et ainsi s'approprier ces relations. Le repreneur doit s'intégrer dans un réseau qu'il n'a pas construit et, pour réussir, il doit s'assurer de sa propre compétence sociale (Baron et Markman, 2003).

\subsubsection{L'évaluation de la ressource réseau (processus de reprise)}

Une fois identifié, le réseau peut être évalué. L'un des résultats de nos observations montrent que seule l'expérience du réseau semble rendre possible son évaluation. En procédant ainsi, le repreneur appréhende la finalité des différentes relations et mesure l'importance de la compétence relationnelle du cédant. En expérimentant le réseau, il peut évaluer l'impact de sa propre compétence relationnelle, a fortiori si le réseau est faiblement «mémorisé» par l'organisation (absence de fichiers, procédures ou d'acteurs du réseau dans l'entreprise autres que le cédant), c'est-à-dire si la capacité de réseautage de l'entreprise est faible (cas 1,2 et Immo). Les cas illustrent la difficulté de l'évaluation de cette ressource lorsque le repreneur potentiel n'est pas encore dans la phase de transition ou lorsque celle-ci est très courte. Le cas 1 montre, par exemple, que l'absence de transition n'a pas permis de faire l'expérience du réseau des fournisseurs par opposition au cas 4 où chaque client a été vu en binôme (cédant/repreneur). Dans le cas de la société Immo, le repreneur a pu observer de manière privilégiée le cédant agir. Deux réseaux distincts ont ainsi été mis au jour. Le premier, d'ordre technique, est facilement identifiable du fait de la régularité des contacts et de l'ancienneté des relations. Son transfert ne semblait pas poser de problèmes car la durée de transition était suffisamment longue pour que les parties se jaugent, s'évaluent et qu'ainsi la confiance se crée. De cette manière, le repreneur pouvait faire montre de ses compétences, de sa bienveillance et de son intégrité tout en testant celles des partenaires et ainsi rendre possible la construction de relation de confiance (Mayer, Davis et Schoorman, 1995). Le second réseau, fait de contacts irréguliers et aléatoires, était plus problématique car il reposait sur le savoir-faire relationnel du cédant. Le transfert de cette ressource était donc hasardeux. Le très haut degré d'implication du cédant dans son entreprise est apparu comme une des clés de la réussite de l'élaboration et de l'utilisation de ce réseau. Cet aspect de l'évaluation a été un frein fort au choix de reprendre cette entreprise. Le repreneur potentiel, jeune, ouvert à d'autres centres d'intérêt, ne se sentait pas prêt à assumer ce même degré de sacrifice (absence de vie de famille, superposition stricte des contacts personnels et professionnels). Nous formulons donc l'hypothèse que seule l'expérience du réseau permet son évaluation, c'est-à-dire la mise au jour des relations de confiance, créatrices de valeur, et la compréhension des 
savoir-faire nécessaires à leur bonne utilisation. L'évaluation du réseau peut être facilitée si celui-ci est un actif de l'organisation et que celle-ci dispose d'une mémoire (p. ex. des fichiers actualisés). L’absence de mémoire de la ressource réseau peut être appréhendée comme un facteur de risque d'échec de la reprise, dans la mesure où l'évaluation de l'entreprise a lieu avant la phase d'entrée dans l'entreprise. Or, nos cas semblent montrer la nécessité d'expérimenter le réseau pour l'évaluer. L'évaluation du réseau est donc à cheval sur les processus de reprise et d'entrée.

\subsubsection{La prise en main de la ressource réseau (processus d'entrée)}

L'entrée dans l'organisation du repreneur, et tout particulièrement la phase de transition, doit être le moment clé de l'utilisation de la ressource réseau (rendue impossible dans le cas 1, exemplaire dans les cas 2 et 4). Dans la phase d'investiture, le repreneur doit logiquement valoriser cette ressource, s'il le peut et s'il le souhaite. Trois types d'utilisation du réseau de l'entreprise reprise ont été observés: 1) l'appropriation. Le repreneur absorbe en l'état la ressource réseau et a la capacité de la mobiliser (cas 3 et 4 pour les fournisseurs);2) l'adjonction, c'est-à-dire que le repreneur associe la ressource réseau reprise à son propre réseau (cas 3 et 4 pour les clients); 3 ) la substitution: le repreneur reconstruit un nouveau réseau car la compétence associée n'est pas transférée (cas 1, cas 2). Le cas Immo permet une observation partielle de cette phase, le repreneur potentiel étant dans les murs mais sans que la reprise soit conclue au moment de l'étude. À l'issue de la phase d'immersion, le repreneur pensait pouvoir transférer le réseau technique parce qu'il avait su créer des relations privilégiées avec les personnes en question et, dans deux cas, avec leurs enfants (successeurs désignés). Il avait constaté qu'il y a un «héritage» possible du réseau. Il pensait avoir les qualités pour animer le réseau d'information mais n'en avait pas l'intention.

\section{TABLEAU 2}

\section{Parallèle entre processus repreneurial et transfert de la ressource réseau}

\begin{tabular}{ll}
\hline Processus repreneurial & Prise en compte de la ressource réseau \\
\hline $\begin{array}{l}\text { Processus de prise de } \\
\text { décision }\end{array}$ & $\begin{array}{l}\text { Perception (être sensible au rôle joué par la ressource } \\
\text { réseau dans le processus de création de valeur d'une firme). }\end{array}$ \\
\hline Processus de reprise & $\begin{array}{l}\text { Évaluation (prise en compte de la valeur effectivement } \\
\text { créé par la ressource réseau, estimation de ses modalités }\end{array}$ \\
\hline & $\begin{array}{l}\text { - compétence relationnelle, mémoire organisationnelle - } \\
\text { son coût de fonctionnement). }\end{array}$ \\
\cline { 2 - 3 } Processus d'entrée & $\begin{array}{l}\text { Utilisation (activation de la ressource; expérimentation } \\
\text { du réseautage). } \\
\text { Valorisation (appropriation, adjonction ou substitution). }\end{array}$ \\
\hline
\end{tabular}




\section{Conclusion}

Quels problèmes pose le transfert de la ressource réseau dans le cadre d'une reprise d'entreprise par une personne physique? À partir de cinq cas, nous proposons une prise en compte spécifique de la ressource réseau en quatre étapes (perception, évaluation, utilisation, valorisation) et ce, pour tout le processus repreneurial. La prise en compte du rôle clé de la ressource réseau doit s'opérer dès le processus de prise de décision afin de rendre possible l'évaluation de ce type d'actif le plus tôt possible dans le processus de reprise. La difficulté de l'évaluation de cette ressource sans en faire l'expérience doit conduire les repreneurs à la prudence en l'absence de mémoire organisationnelle dédiée et les inciter à s'assurer que le transfert de la ressource réseau se fasse lors de la phase de transition.

L'observation de la prise en main effective de la ressource réseau semble donner lieu à des formes de valorisation variables (appropriation/ substitution). Cet aspect de la recherche nécessite des approfondissements. En effet, la substitution est-elle toujours un échec? Peut-elle être délibérée? Quelle est l'évolution dans le temps de la ressource réseau?

Nos résultats revêtent un caractère hypothétique conformément à la logique abductive et nécessitent confirmation. Dans le cas de la recherche exploratoire, l'observation repose uniquement sur la vision du repreneur, ce qui est une limite. D'autres études de cas longitudinales seraient un complément précieux (incluant le cédant et les parties prenantes). Par ailleurs, la portée des résultats doit être limitée au champ de l'artisanat et de la très petite entreprise. Des travaux portant sur des PME plus structurées sont donc envisageables.

\section{Bibliographie}

ARRÈGLE, J.-L. (1995), L'identification des actifs stratégiques, premières étapes de l'analyse «resource-based», Nice, IAE, RODIGE, Cahier de recherche 95-01, 21 p.

BARON, R. et G. MARKMAN (2003), «Beyond social capital: the role of entrepreneurs' social competence in their financial success », Journal of Business Venturing, vol. 18, p. 41-60.

Bourdieu, P. (1980), «Le capital social», Actes de la recherche en sciences sociales, $\mathrm{n}^{\circ} 31$.

BRADACH, J. et R. ECCLES (1989), «Price, authority and trust: from ideal types to plural forms », Annual Review of Sociology, vol. 15, p. 97-118.

BrouARD, F. et L. CADIEUX (2007), «La transmission des PME: vers une meilleure compréhension du contexte», Communication soumise à l'Académie de l'Entrepreneuriat, Sherbrooke, 4-5 octobre, 22 p. 
BURT, R. (1995), «Le capital social, les trous structuraux et l'entrepreneur », Revue française de sociologie, vol. XXXVI, p. 599-628.

CAdieuX, L. (2005), «La succession dans les PME familiales: proposition d'un modèle de réussite du processus de désengagement du prédécesseur », Revue internationale PME, vol. 18, nºs 3-4, p. 31-49.

Charreire, S. et F. Durrieux (1999), «Explorer et tester», dans R.A. Thiétart (dir.), Méthodes de recherche en management, Paris, Dunod, p. 57-80.

Coleman, J. (1988), «Social capital in the creation of human capital», American Journal of Sociology, vol. 94, p. 95-120.

DAVIDSON, P. et B. HONIG (2003), «The role of social and human capital among nascent entrepreneurs », Journal of Business Venturing, vol. 18, p. 301-331.

DESCHAMPS, B. (2002), «Les spécificités du processus repreneurial », Revue française de gestion, vol. 28, $\mathrm{n}^{\circ} 138$.

Dussuc, B. et S. GEINDRE (2000), «Comment les PME utilisent leur réseau pour se financer? Une réflexion à partir de quatre cas », dans T. Durand, E. Mounoud et G. Koenig (dir.), Perspectives en management stratégique T6, Caen, Éditions Management et Société, p. 77-102.

EISENHARDT, K. (1989), «Building theories from case study research », Academy of Management Review, vol. 14, no 4, p. 532-550.

FÉDÉRATION CANADIENNE DE L'ENTREPRISE INDÉPENDANTE - FCEI (2005), «La relève: la clé de la réussite», document de recherche, <http://www.cfib.ca/ researchf/reports/rr3007f.pdf>.

FROEHLICHER, T. (1997), Éléments sur le management des coopérations interentreprises: une contribution en termes de configurations relationnelles, Thèse de doctorat en sciences de gestion, Université de Nancy II.

Glaser, B.G. (1978), Theoretical Sensitivity: Advances in the Methodology of Grounded Theory, Mill Valley, The Sociology Press.

Glaser, B.G. et L. STRAuSs (1967), The Discovery of Grounded Theory: Strategies for Qualitative Research, New York, Aldine de Gruyter.

GranovetTER, M. (1985), «Economic action and social structure: the problem of embeddedness », American Journal of Sociology, vol. 91, n 3, p. 481-510.

GRANT, R. (1991), «The resource-based theory of competitive advantage: implications for strategy formulation», California Management Review, vol. 33, n 3, p. $114-135$

Gulati, R., N. NOHRIA et A. ZAHEER (2000), «Strategic networks », Strategic Management Journal, vol. 21, mars, p. 203-215.

HALL, R. (1992), «The strategic analysis of intangible resources », Strategic Management Journal, vol. 13, p. 135-144.

KPMG (2008), «La transmission des entreprises industrielles, un enjeu plus fort en France qu'en Europe», Étude pour le ministère de l'Économie, de l'Industrie et de l'Emploi (MEIE). 
LIN, N (1999), «Building a network theory of social capital», Connections, vol. 22, $\mathrm{n}^{\circ} 1, \mathrm{p} .28-51$.

MARCHESNAY, M. et P.-A. JULIEN (1990), «The small business as a transaction space», Entrepreneurship and Regional Development, vol. 2, no 3, p. 267-278.

MAYER, R., J. DAVIS et F. SCHOORMAN (1995), «An integrative model of organizational trust », Academy of Management Review, vol. 20, n 3, p. 709-734.

PiCARD, C. et C.THÉVENARD-PUTHOD (2004), «La reprise de l'entreprise artisanale : spécificités du processus et conditions de sa réussite», Revue internationale $P M E$, vol. 17, $\mathrm{n}^{\circ}$ 2, p. 93-121.

Powell, W. (1987), «Hybrid organizational arrangements : new form or transitional development?», California Management Review, vol. XXX, n 1, p. 67-87.

Powell, W. (1990), «Neither market nor hierarchies: networks forms of organization », Research in Organizational Behavior, vol. 12, p. 295-336.

$\mathrm{SOH}, \mathrm{P} .(2003)$, «The role of networking in information acquisition and its implications for new product performance », Journal of Business Venturing, vol. 18, p. 727-744.

ST-CYR, L. et F. RICHER (2005), «La planification du processus de transmission dans les PME québécoise», Revue internationale PME, vol. 18, ns 3-4, p. 51-71.

SUIRE, R. (2004), «Des réseaux de l'entrepreneur aux ressorts du créatif: quelles stratégies pour les territoires », Revue internationale PME, vol. 17, n 2, p.123-143.

TeEce, D., G. PisAno et A. Shuen (1997), «Dynamic capabilities and strategic management», Strategic Management Journal, vol. 18, n 7, p. 509-533.

Thorelli, H. (1986), «Networks : between markets and hierarchies», Strategic Management Journal, vol. 7, p. 37-51.

TYWONIAK, S. (1998) «Le modèle des ressources et des compétences: un nouveau paradigme pour le management stratégique? », dans H. Laroche et J.-P. Nioche (dir.), Repenser la stratégie, Paris, Vuibert, p. 166-204.

WAlter, A., M. Auer et T. RitTER (2006), «The impact of network capabilities and entrepreneurial orientation on university spin-off performance», Journal of Business Venturing, vol. 21, p. 541-567.

WERnERFELT, B. (1984), «A resource-based view of the firm », Strategic Management Journal, vol. 5, p. 171-180.

Williamson, O.E. (1985), The Economic Institutions of Capitalism, Firms Markets, Relational Contracting, New York, Free Press.

YIN, R.K. (1989), Case Study Research. Design and Methods, Londres, Sage Publications, $166 \mathrm{p}$. 